\title{
Placental biometry for prediction of small for gestational age fetuses in low resource setting
}

\author{
Megha Jindal $^{1 *}$, Sangeeta Gupta ${ }^{2}$
}

\begin{abstract}
${ }^{1}$ Department of Obstetrics and Gynecology, University College of Medical Sciences and Associated Hospital, Delhi, India

${ }^{2}$ Department of Obstetrics and Gynecology, Maulana Azad Medical College and Associated Hospitals, Delhi, India
\end{abstract}

Received: 24 October 2017

Accepted: 28 October 2017

\author{
*Correspondence: \\ Dr. Megha Jindal, \\ E-mail: jindal.megha@gmail.com
}

Copyright: () the author(s), publisher and licensee Medip Academy. This is an open-access article distributed under the terms of the Creative Commons Attribution Non-Commercial License, which permits unrestricted non-commercial use, distribution, and reproduction in any medium, provided the original work is properly cited.

\section{ABSTRACT}

Background: Small for gestational age refers to foetuses with birth weight less than tenth centile for gestational age. Such foetuses are at increased risk of intrauterine fatal demise in comparison to others. Placenta plays a central role in supporting foetal growth. Researchers have emphasized on three dimensional sonographic placental volumetry as a predictor of SGA. This study focussed on role of two dimensional Ultrasonographic placental measurement in predicting SGA foetuses.

Methods: Prospective study was conducted at Department of Obstetrics and Gynecology, Maulana Azad Medical College from November 2013 to February 2015. In singleton pregnancies at 18-22 weeks of gestation, placental biometry (in two dimensions) was performed. Maximal Placental Diameter (MaxPD) and Maximal Placental Thickness (MaxPT) in two orthogonal planes was recorded. Mean Placental Diameter (MPD) and Mean Placental Thickness (MPT) were calculated. At the time of delivery, as per the birth weight the neonate was classified into appropriate for gestational age (AGA)/ SGA/ large for gestational age (LGA). MPD and MPT were analyzed as predictors of SGA.

Results: Both the MaxPDs and MPD were significantly smaller in SGA pregnancies (all with $p \leq 0.001$ ). Similarly, both the MaxPTs $(p=0.006$ and $p=0.001)$ and MPT $(p=0.000)$ were significantly smaller in SGA pregnancies. The ROC curve for combined placental biometry had the maximum area under the curve $(0.805)$.

Conclusions: Placental measurements taken in mid-gestation are a valuable predictor of SGA. Measurement of placental diameter and thickness is quick and simple. This approach should be explored in future to develop a predictive model for growth restricted foetuses.

Keywords: Placental biometry, Small for gestational age fetuses

\section{INTRODUCTION}

Small for gestational age (SGA) refers to foetuses with birth weight less than tenth centile for gestational age. ${ }^{1}$ Worldwide, the prevalence of SGA is $27 \%$ of live births, whereas it is $46.9 \%$ in India alone. ${ }^{2}$ The risk of intrauterine foetal death is more in SGA versus non-SGA at all gestational ages. Further, foetuses with lower centile threshold have greater risk of intrauterine foetal death. ${ }^{3}$ Unfortunately, only 50\% growth restricted foetuses are timely identified in antenatal period. ${ }^{4}$

The placenta plays a central role in supporting foetal growth. Researchers have emphasized the importance of three dimensional sonographic placental volumetry as an indicator of SGA. ${ }^{5}$ However, in comparison to the twodimensional placental measurements, three dimensional measurements are more complex, time consuming, 
require greater expertise and not widely available; thus, limiting their clinical utility as a routine test. ${ }^{6}$

This study was therefore designed to evaluate the role of two dimensional ultrasonographic placental measurements in early and timely prediction of SGA foetuses. To the best of my knowledge, there is no existing data from Indian population in this context.

\section{METHODS}

Patients with singleton live pregnancy attending the antenatal Outpatient Department of Obstetrics and Gynecology, Lok Nayak Hospital between 18 weeks 0 days and 22 weeks 6 days period of gestation from November 2013 to February 2015 were recruited.

Women with multiple gestation or with medical illness such as chronic hypertension, chronic renal disease, or diabetes mellitus or with congenitally malformed fetus were excluded. Pregnancies were dated by last menstrual period in case the patient was sure of dates or by either first trimester or early second trimester ultrasonography up to 16 weeks.

Fetal and placental biometry was performed on all the patients using Philips HD7 ultrasound machine with transabdominal probe of 2 to $5 \mathrm{MHz}$.

\section{Fetal biometry}

The following fetal biometric parameters were recorded in terms of gestational age: head circumference (HC), biparietal diameter (BPD), abdominal circumference (AC), femur length (FL), transverse cerebellar diameter (TCD), and estimated fetal weight (EFW) using Hadlock's formula. $^{7}$

\section{Placental biometry}

Placental biometry was done in the same manner as done by Schwartz et al in their study titled "Two-dimensional sonographic placental measurements in the prediction of SGA infants.",

- Placenta was scanned from various angles to obtain the largest placental diameter possible-Maximal Placental Diameter (MaxPD). Then, the diameter was measured along the fetal surface using a linear or bilinear approach (whichever is deemed a better fit) as diagrammatically represented in Figure 1.

- In the same image, the Maximal Placental Thickness (MaxPT) was recorded as shown in Figure 1.

- Now, the ultrasound probe was rotated by $90^{\circ}$ and the above measurements were repeated in this orthogonal plane.

- Using the two values obtained, the Mean Placental Diameter (MPD) and Mean Placental Thickness (MPT) were calculated.

\section{Follow up}

Routine antenatal care was provided to the patients. Management and decision for delivery was taken as per the hospital protocols. Clinical management of the individual pregnancies including use of steroids, tocolysis, additional ultrasounds or iatrogenic premature delivery was left to the attending obstetrician. After the delivery, gestational age at delivery, mode of delivery, the birth weight of the neonate and placental weight were recorded. The neonate was classified into: AGA (appropriate for gestational age), SGA (small for gestational age) with birth weight $<10^{\text {th }}$ centile for gestational age and LGA (large for gestational age) with birth weight $>90^{\text {th }}$ centile for gestational age. ${ }^{9}$

Results were analyzed using Statistical Package for the Social Sciences (SPSS) statistical software package (SPSS Inc., Chicago, IL, version 17.0 for windows).

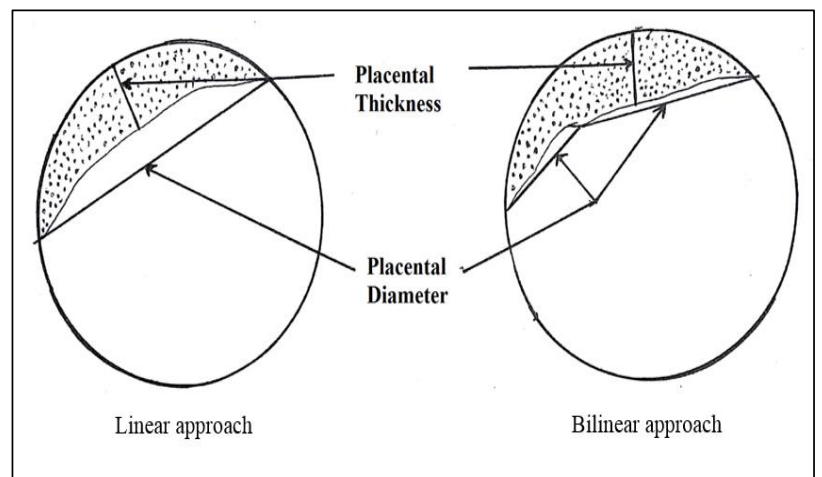

The dotted portion represents placenta. Linear and bilinear approach of placental measurement has been shown (Adapted from Schwartz N, 2012 ${ }^{8}$ ).

Figure 1: Diagrammatic representation of methods of measuring MaxPD.

\section{RESULTS}

During the study period, 426 pregnant women attending the antenatal clinic in Department of Obstetrics and Gynecology, Maulana Azad Medical College and Lok Nayak Hospital were recruited for the study as per the inclusion criterion. However, data of 423 subjects was analyzed as three patients, who had home delivery, were excluded from the analysis due to non-availability of baby weight and placental weight at delivery.

A second trimester anomaly scan was routinely performed in all the patients at 18-20 weeks of gestation. None of the foetus in the study group had any congenital malformations.

Based on the birth weight centiles of the neonate according to gestational age, the study group was divided into 3 groups: AGA, SGA and LGA. Amongst 423 women; $362(85.6 \%)$ delivered AGA neonates, 33 (7.8\%) 
delivered SGA neonates and $28(6.6 \%)$ delivered LGA neonates.
The MPD in AGA group was $12.87 \pm 1.48 \mathrm{~cm}$, in SGA group was $11.51 \pm 1.18 \mathrm{~cm}$ and in LGA group was $14.15 \pm 1.61 \mathrm{~cm}$ (Table 1a).

Table 1a: Placental biometry in the study subgroups (placental diameter).

\begin{tabular}{|c|c|c|c|c|c|}
\hline Placental biometry & Group & Number (n) & Mean (cm) & Standard deviation (cm) & Range (cm) \\
\hline \multirow{3}{*}{ Max PD ( $1^{\text {st }}$ plane $)$} & AGA & 362 & 12.94 & 1.61 & $8.46-17.95$ \\
\hline & SGA & 33 & 11.43 & 1.47 & $8.21-14.86$ \\
\hline & LGA & 28 & 14.28 & 1.79 & $9.88-16.80$ \\
\hline \multirow{3}{*}{ Max PD ( $2^{\text {nd }}$ plane $)$} & AGA & 362 & 12.80 & 1.67 & $7.10-18.93$ \\
\hline & SGA & 33 & 11.58 & 1.34 & $9.30-14.40$ \\
\hline & LGA & 28 & 14.03 & 1.74 & $11.00-16.96$ \\
\hline \multirow{3}{*}{ Mean PD } & AGA & 362 & 12.87 & 1.48 & $8.70-18.44$ \\
\hline & SGA & 33 & 11.51 & 1.18 & $9.74-14.05$ \\
\hline & LGA & 28 & 14.15 & 1.61 & $10.69-16.64$ \\
\hline
\end{tabular}

Table 1b: Placental biometry in the study subgroups (placental thickness).

\begin{tabular}{|c|c|c|c|c|c|}
\hline Placental biometry & Group & Number (n) & Mean $(\mathrm{cm})$ & Standard deviation $(\mathrm{cm})$ & Range (cm) \\
\hline \multirow[t]{3}{*}{ Max PT (1 ${ }^{\text {st }}$ plane $)$} & AGA & 362 & 3.05 & 0.76 & $1.30-9.28$ \\
\hline & SGA & 33 & 2.63 & 0.57 & $1.53-3.70$ \\
\hline & LGA & 28 & 3.17 & 0.60 & $1.82-4.11$ \\
\hline \multirow[t]{3}{*}{ Max PT ( $2^{\text {nd }}$ plane $)$} & AGA & 362 & 2.98 & 0.69 & $0.64-5.59$ \\
\hline & SGA & 33 & 2.52 & 0.49 & $1.34-3.80$ \\
\hline & LGA & 28 & 3.33 & 0.76 & $2.21-4.64$ \\
\hline \multirow[t]{3}{*}{ Mean PT } & AGA & 362 & 3.02 & 0.62 & $1.45-5.96$ \\
\hline & SGA & 33 & 2.58 & 0.42 & $1.84-3.35$ \\
\hline & LGA & 28 & 3.25 & 0.61 & $2.12-4.30$ \\
\hline
\end{tabular}

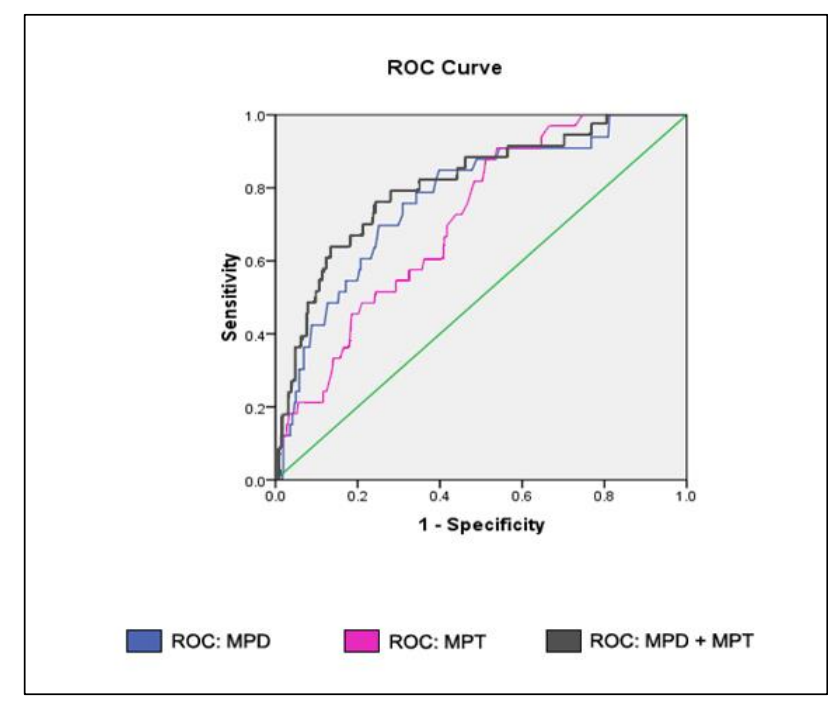

Figure 2: ROC curve for placental biometry.

The MPT in AGA group was $3.02 \pm 0.62 \mathrm{~cm}$, in SGA group was $2.58 \pm 0.42 \mathrm{~cm}$ and in LGA group was $3.25 \pm 0.61 \mathrm{~cm}$ (Table 1b). Both the MaxPDs and MPD were significantly greater in AGA pregnancies than in SGA pregnancies (all had $\mathrm{p} \leq 0.001$ ). Similarly, both the MaxPTs $(p=0.006$ and $p=0.001)$ and MPT $(p=0.000)$ were statistically greater in AGA group than in SGA group.

The Receiver Operator Characteristics (ROC) curves were generated for MPD, MPT and the combination of these two parameters in prediction of SGA as shown in Figure 2. The area under curve (AUC) is shown in Table 2. The AUC for each of the three curves was found to be significant. The ROC curve for combined placental biometry (MPD and MPT) had the maximum AUC as shown in Figure 2, signifying the combination of the two parameters is better than either of them performed alone, for the prediction of SGA.

Based on the ROC curve, for mean placental diameter a cut off value of $<12.56 \mathrm{~cm}$, at which maximum sensitivity of $84.85 \%$ and maximum specificity of $60.22 \%$, has been set. For mean placental thickness a cut off value of $<3.05 \mathrm{~cm}$ has been decided, with maximum sensitivity of $90.91 \%$ and maximum specificity of 46.13\%. Sensitivity, specificity, positive predictive value (PPV) and negative predictive value (NPV) of placental measurements smaller than the cut-off were determined for occurrence of SGA. The PPV and specificity of the combined biometry was found to be greater than either of them alone (Table 3). 
Table 2: Area under curve (AUC).

\begin{tabular}{|llll|}
\hline Tests & AUC & $\begin{array}{l}95 \% \text { Confidence } \\
\text { intervals }\end{array}$ & P value \\
\hline MPD & 0.772 & $0.690-0.853$ & 0.000 \\
\hline MPT & 0.713 & $0.635-0.791$ & 0.000 \\
\hline MPD + MPT & 0.805 & $0.725-0.884$ & 0.000 \\
\hline
\end{tabular}

Table 3: Sensitivity, specificity, positive predictive value (PPV), negative predictive value (NPV) for prediction of SGA.

\begin{tabular}{|lllll|}
\hline Test & $\begin{array}{l}\text { Sensitivity } \\
(\%)\end{array}$ & $\begin{array}{l}\text { Specificity } \\
(\%)\end{array}$ & $\begin{array}{l}\text { PPV } \\
(\%)\end{array}$ & $\begin{array}{l}\text { NPV } \\
(\%)\end{array}$ \\
\hline $\begin{array}{l}\text { MPD } \\
<12.56 \mathrm{~cm}\end{array}$ & 84.85 & 60.22 & 16.28 & 97.76 \\
\hline $\begin{array}{l}\text { MPT } \\
<3.05 \mathrm{~cm}\end{array}$ & 90.91 & 46.13 & 13.33 & 98.24 \\
\hline $\begin{array}{l}\text { MPD } \\
<12.56 \mathrm{~cm}\end{array}$ & 81.82 & 74.31 & 22.50 & 97.82 \\
\hline $\begin{array}{l}\text { +MPT } \\
<3.05 \mathrm{~cm}\end{array}$ & & & & \\
\hline
\end{tabular}

\section{DISCUSSION}

Early reliable predictors of placental dysfunction remain lacking in obstetric care. It is likely that no single test will achieve sufficient accuracy to be used in clinical practice as a stand-alone test in the prediction of SGA. Currently, only $50 \%$ cases of fetal growth restriction get diagnosed correctly in the antenatal period. ${ }^{8}$ Accurate identification of fetal growth impairment leads to four times reduction in neonatal complications and death. ${ }^{10}$ Administration of low dose aspirin before 16 weeks gestation leads to significant reduction in fetal growth restriction. ${ }^{11}$

Protocols for screening strategies do not exist currently for identification of pregnancies at risk of serious uteroplacental insufficiency. However, the present body of evidence from both biochemistry and ultrasound data suggests that a proportion of SGA pregnancies can be recognized using these tools. ${ }^{12}$ The present study evaluates the effect of two-dimensional second trimester placental biometry in predicting women at risk of placental insufficiency. To the best of my knowledge, no study in this context has been conducted so far in the Indian population.

Graafmans et al has stated that to improve the identification of growth restricted infants, population specific standards for birth weight should be developed and used. ${ }^{13}$. The variation in the incidence of SGA for the same population may be attributed to the differences in the reference population used to classify SGA and differences in the use of customized or non-customized charts. In this study, growth chart given by Singh $M$ to classify newborn into AGA, SGA and LGA was used. ${ }^{9}$ Singh $M$ reference population has been developed on neonatal weights in Indian population.
The ultrasound was performed in the second trimester at 18 weeks 0 days to 22 weeks 6 days of gestation. The timing of scan was based on the fact that a discrete placenta gets formed by 16 - 18 weeks with completion of vascular remodeling of uterine spiral arteries. The mean gestational age at the time of scan in AGA group was $20.52 \pm 0.98$ weeks, in SGA group was $20.52 \pm 0.93$ weeks and in LGA group was $20.73 \pm 0.91$ weeks. The mean gestational age at the time of scan between AGA and SGA group was comparable, $\mathrm{p}=1.000$.

The principal determinant of birth weight is the transfer efficiency of placental nutrients and oxygen, a mechanism highly dependent on a well grown placenta. ${ }^{14}$ Thus, focusing placental evaluation, 2D placental biometry was performed. The maximal diameter and thickness of the placenta were recorded in the two orthogonal planes based on several considerations. First, this method mimics the standard two diameters measured on the delivered placentae sent for pathology evaluation. Second, placental shape often deviates from a perfect circle. Thus, a single maximal diameter may not be sufficiently descriptive. Third, rotation of the transducer by $90^{\circ}$ gives the opportunity to capture the third dimension without the need for three-dimensional imaging equipment. Placental diameter was measured on the fetal surface of the placenta, in contrast to its measurement midway between the two placental surfaces by other investigators. This method had the following advantages. First, fetal surface could be easily identified due to contrast between the echogenic placenta and anechoic amniotic fluid. Second, the concavity of the fetal surface made it more likely for the placental edges to fall within the sector width of the ultrasound probe. Schwartz et al also performed the placental measurements (diameter and thickness) at similar gestation, 18-24 weeks' gestation. ${ }^{8}$

The MPD in AGA group is $12.87 \pm 1.48 \mathrm{~cm}$, in SGA group is $11.51 \pm 1.18 \mathrm{~cm}$ and in LGA group is $14.15 \pm 1.61$ $\mathrm{cm}$. The MPT in AGA group is $3.02 \pm 0.62 \mathrm{~cm}$, in SGA group is $2.58 \pm 0.42 \mathrm{~cm}$ and in LGA group is $3.25 \pm 0.61$ $\mathrm{cm}$. All the placental measurements i.e. both the MaxPDs $(p \leq 0.001)$, MPD $(p \leq 0.001)$, both the MaxPTs $(p=0.006$ and $p=0.001)$ and MPT $(p=0.000)$ are statistically more in AGA group than in SGA group. Thus, the study showed definite association between simple twodimensional placental measurements and subsequent delivery of SGA neonate. These results were similar to the findings of the study by Schwartz et al according to which MPD ( $p<0.001)$ and MPT ( $p$ <0.006) were significantly smaller in SGA pregnancies. ${ }^{8}$

ROC curves were generated for MPD and MPT for prediction of SGA, MPD (AUC 0.772) performed better than MPT (AUC 0.713) in the prediction of SGA. Inclusion of MPD and MPT into a combined model to predict SGA yielded a slightly higher AUC (0.805) than MPD (AUC 0.772) and MPT (AUC 0.713) alone. This was similar to findings of Schwartz et al. ${ }^{8}$ Thus, mid- 
gestation placental biometry on two-dimensional scan proved to be an important predictor of SGA.

Based on the ROC curve, for MPD a cut off value of $<12.56 \mathrm{~cm}$ was chosen at which maximum sensitivity of $84.85 \%$ and maximum specificity of $60.22 \%$ were attained. Similarly, for MPT a cut off value of $<3.05 \mathrm{~cm}$ was chosen at which maximum sensitivity of $90.91 \%$ and maximum specificity of $46.13 \%$ were attained. On combining MPD and MPT at the above-mentioned cut offs sensitivity of $81.82 \%$, specificity of $74.31 \%$, PPV of $22.50 \%$ and NPV of $97.82 \%$ were attained. Though the PPV was less, but the high NPV of the placental biometry gives the advantage of reasonably ruling out an adverse outcome.

Placental biometry measurement by two-dimensional ultrasonography has innumerable advantages ranging from simple inexpensive technique, easily available equipment, less expertise, quick evaluation and interoperator reproducibility. Further, the placental measurements can be integrated with anatomical ultrasound scan in the second trimester, thus decreasing the number of antenatal visits of the patient and increasing the compliance.

The strength of this study lies in its prospective evaluation of the second trimester placental biometry for prediction of SGA in a reasonable sample size. The study subjects form a low-risk cohort. Majority of the researchers have targeted high risk population whereas our study throws light on the pattern of alteration in placental biometry in low-risk population. ${ }^{15-19}$ All the ultrasounds were solely performed by single examiner thus, eliminating the inter-observer variability in assessment. The attending obstetrician was blinded to the second trimester ultrasound findings and hence, had no influence on the outcome.

However, there are few limitations as well. All the study subjects did not have first trimester scan to confirm the gestational age. However, on Ballard's score all neonates corresponded to the period of gestation as per the last menstrual period or the early scan of upto 16 weeks gestation. Demographic variables such as maternal height, weight, prep-pregnancy BMI, previous obstetric history was not included in predictive models for SGA in this study.

Customized growth charts to classify neonates in AGA and SGA were not used, instead population based charts given by Singh $\mathrm{M}$ were utilized. ${ }^{12}$ Though SGA is a commonly used primary outcome in Obstetrics, it fails to distinguish between constitutional smallness and pathological growth restriction. Perinatal outcome of the neonate should have been evaluated in terms of Apgar score, umbilical artery $\mathrm{pH}$, NICU admission, neonatal death-early and late, growth and development, achievement of milestones and neurodevelopment outcome later in life.
We propose that women with abnormal second trimester placental biometry should have monitoring of fetal growth velocity.

\section{CONCLUSION}

Placental measurements taken in mid-gestation are a valuable predictor of SGA. Measurement of placental diameter and thickness is quick and simple. This approach should be explored in future to develop a predictive model for growth restricted foetuses.

Funding: No funding sources

Conflict of interest: None declared

Ethical approval: The study was approved by the Institutional Ethics Committee

\section{REFERENCES}

1. WHO. Physical status: the use and interpretation of anthropometry. Report of a WHO Expert Committee. World Health Organ Tech Rep Ser. 1995;854:1-452.

2. Lee AC, Katz J, Blencowe H, Cousens S, Kozuki N, Vogel JP, et al. National and regional estimates of term and preterm babies born small for gestational age in 138 low-income and middle-income countries in 2010. Lancet Glob Health. 20131(1):e26-36.

3. Pilliod RA, Cheng YW, Snowden JM, Doss AE, Caughey AB. The risk of intrauterine fetal death in the small-for-gestational-age fetus. Am J Obstet Gynecol. 2012;207(4):318.e1-6.

4. American College of Obstetricians and Gynecologists (ACOG) Practice bulletin no. 134: fetal growth restriction. Obstet Gynecol. 2013;121(5):1122-33.

5. Schwartz N, Coletta J, Pessel C, Feng R, TimorTritsch IE, Parry S, et al. Novel 3-dimensional placental measurements in early pregnancy as predictors of adverse pregnancy outcomes. J Ultrasound Med. 2010;29(8):1203-12.

6. Campbell S. Is placental size a good predictor of obstetric complications?. Ultrasound Obstet Gynecol 2009;34(3):247-8.

7. Hadlock FP, Harrist RB, Sharman RS, Deter RL, Park SK. Estimation of fetal weight with the use of head, body, and femur measurements--a prospective study. Am J Obstet Gynecol. 1985;151(3):333-7.

8. Schwartz N, Wang E, Parry S. Two-dimensional sonographic placental measurements in the prediction of small-for-gestational-age infants. Ultrasound Obstet Gynecol. 2012;40(6):674-9.

9. Singh M. Pediatric clinical methods. Examination of a newborn baby. $3^{\text {rd }}$ ed. New Delhi: Sagar Publications; 2009; 14:242.

10. Albu AR, Anca AF, Horhoianu VV, Horhoianu IA. Predictive factors for intrauterine growth restriction. J Med Life. 2014;7(2):165-71.

11. Roberge S, Nicolaides KH, Demers S, Villa P, Bujold E. Prevention of perinatal death and adverse perinatal outcome using low-dose aspirin: a meta- 
analysis. Ultrasound Obstet Gynecol. 2013;41(5):491-9.

12. Whittle W, Chaddha V, Wyatt P, Huppertz B, Kingdom J. Ultrasound detection of placental insufficiency in women with unexplained abnormal maternal serum screening results. Clin Genet. 2006;69:97-104.

13. Graafmans WC, Richardus JH, Borsboom GJ, Bakketeig L, Langhoff-Roos J, BergsjøP, et al. Birth weight and perinatal mortality: a comparison of optimal birth weight in seven Western European countries. Epidemiol. 2002;13(5):569-74.

14. Misra DP, Salafia CM, Miller RK, Charles AK. Nonlinear and gender-specific relationships among placental growth measures and the fetoplacental weight ratio. Placenta. 2009;30(12):1052-7.

15. Proctor LK, Toal M, Keating S, Chitayat D, Okun N, Windrim RC, et al. Placental size and the prediction of severe early-onset intrauterine growth restriction in women with low pregnancy-associated plasma protein-A. Ultrasound Obstet Gynecol. 2009;34(3):274-82.

16. Toal M, Chaddha V, Windrim R, Kingdom J. Ultrasound detection of placental insufficiency in women with elevated second trimester serum alphafetoprotein or human chorionic gonadotropin. J Obstet Gynecol Can. 2008;30(3):198-206.

17. Costa SL, Proctor L, Dodd JM, Toal M, Okun N, Johnson JA, et al. Screening for placental insufficiency in high-risk pregnancies: is earlier better?. Placenta. 2008;29(12):1034-40.

18. Toal M, Chan C, Fallah S, Alkazaleh F, Chaddha V, Windrim RC, et al. Usefulness of a placental profile in high-risk pregnancies. Am J Obstet Gynecol. 2007;196(4):363.e1-7.

19. Viero S, Chaddha V, Alkazaleh F, Simchen MJ, Malik A, Kelly E, et al. Prognostic value of placental ultrasound in pregnancies complicated by absent end-diastolic flow velocity in the umbilical arteries. Placenta. 2004;25(8-9):735-41.

Cite this article as: Jindal M, Gupta S. Fetal Placental biometry for prediction of small for gestational age fetuses in low resource setting. Int $\mathrm{J}$ Reprod Contracept Obstet Gynecol 2017;6:5266-71. 